\title{
Multiscale reconstruction for computational spectral imaging
}

\author{
R. M. Willett ${ }^{a}$, M. E. Gehm ${ }^{b}$, and D. J. Brady ${ }^{c}$ \\ ${ }^{a}$ Department of Electrical and Computer Engineering, Duke University, Durham, NC 27708 \\ ${ }^{b}$ Department of Electrical and Computer Engineering, University of Arizona, Tucson AZ, 85721 \\ ${ }^{a}$ Fitzpatrick Institute for Photonics, Duke University, Durham, NC 27708
}

\begin{abstract}
In this work we develop a spectral imaging system and associated reconstruction methods that have been designed to exploit the theory of compressive sensing. Recent work in this emerging field indicates that when the signal of interest is very sparse (i.e. zero-valued at most locations) or highly compressible in some basis, relatively few incoherent observations are necessary to reconstruct the most significant non-zero signal components. Conventionally, spectral imaging systems measure complete data cubes and are subject to performance limiting tradeoffs between spectral and spatial resolution. We achieve single-shot full 3D data cube estimates by using compressed sensing reconstruction methods to process observations collected using an innovative, real-time, dual-disperser spectral imager. The physical system contains a transmissive coding element located between a pair of matched dispersers, so that each pixel measurement is the coded projection of the spectrum in the corresponding spatial location in the spectral data cube. Using a novel multiscale representation of the spectral image data cube, we are able to accurately reconstruct $256 \times 256 \times 15$ spectral image cubes using just $256 \times 256$ measurements.
\end{abstract}

Keywords: wavelets, compressed sensing, hyperspectral imaging

\section{COMPRESSIVE SPECTRAL IMAGING}

Spectral imaging is a highly effective tool in a variety of scientific and engineering contexts because of the information it implicitly encodes about the nature of the materials being imaged. While traditional digital imaging techniques produce images with scalar values associated with each spatial pixel location, in spectral images these scalar values are replaced with a vector containing the spectral information from that spatial location. The resulting data is therefore three-dimensional (two spatial dimensions and one spectral dimension). Spectral information can be vital for tasks such as monitoring the health of a forest ecosystem [1,2], increasing our understanding of solar physics [3], or examining tissue and organisms used to study cellular dynamics [4,5].

Despite its potential, however, many modern spectral imagers face a limiting tradeoff between spatial and spectral resolution, with the total number of voxels measured constrained by the size of the detector array. This constraint limits the utility and cost-effectiveness of spectral imaging for many applications. Furthermore, straightforward application of traditional spectroscopic techniques to spectral imaging proves problematic. The simplest spectral imager forms combine either a pushbroom (linear scanning) or tomographic (rotational scanning) front-end with a traditional slit-based dispersive spectrometer. Unfortunately, in many of the most interesting applications (most notably biophotonics) the sources are often both spatially-incoherent and weak. Spatially-incoherent sources present a significant challenge to slit-based dispersive spectrometers, and extremely poor photon collection efficiency is the result. When the source is also weak, the absolute number of collected

Send correspondence to willett@ duke.edu. R. Willett was supported by Defense Advanced Research Projects Agency grants HR0011-06-1-0026 and N00014-06-1-0610. D. Brady and M. Gehm were supported by Defense Advanced Research Projects Agency grant N00014-06-1-0610.

Computational Imaging $\mathrm{V}$, edited by Charles A. Bouman, Eric L. Miller, llya Pollak Proc. of SPIE-IS\&T Electronic Imaging, SPIE Vol. 6498, 64980L, @ 2007 SPIE-IS\&T · 0277-786X/07/\$18 
photons can be very small. Because the small number of photons must then be apportioned between the large number of voxels in the data cube, a given spatio-spectral element tends to contain very few photons and hence has a poor signal-to-noise ratio (SNR).

This paper addresses these challenge by presenting a novel spectral imaging system and associated reconstruction framework. The imager is a completely static, single-shot design, resulting in a mechanically robust and inexpensive system. One critical design element is that the imager does not directly measure each voxel in the desired three-dimensional spectral image. Instead, it collects a small number (relative to the size of the data cube) of coded measurements, and then a novel reconstruction method is used to estimate the spectral image from the noisy projections.

As indicated by recent work in the emerging field of compressed sensing [6-8], when feasible, judicious selection of the type of distortion induced by measurement systems may dramatically improve our ability to extract high-quality signals from a limited number of measurements. The basic idea of this theory is that when the signal of interest is very sparse (i.e. zero-valued at most locations) or highly compressible in some basis, relatively few incoherent observations are necessary to reconstruct the most significant non-zero signal components. This paper presents a practical application of these ideas to spectral imaging, and demonstrates that accurate spectroscopic images can be estimated from the noisy projections collected by the proposed system.

In Section 2, we present our spectral imaging system design and its advantages over conventional systems. We then describe the computational methods used to estimate the spectral image from noisy measurements collected by the proposed system. First we focus on noise removal from spatio-spectral data cubes in Section 3, and then we describe how this denoising framework can be used to solve the highly ill-posed inverse problem associated with our imaging system in Section 4. Results are presented in Section 5, and discussed in Section 6.

\section{IMAGING SYSTEM DESIGN}

\subsection{Design motivation}

Over the years, many groups have addressed the light collection problem associated with spectral imaging, and have produced a number of creative solutions. Two very advanced methods are the scanning-Michelson Fourier-transform spectrometers, and multiplexed pushbroom designs based on digital micro-mirror (DMM) technology $[9,10]$. Both approaches have proven highly successful in simulation and experiment, however they rely on mechanical systems that are simultaneously expensive and fragile.

Other groups have focused on simpler schemes that are more robust and inexpensive, most notably several direct-view designs that maximize the light gathering efficiency of the systems [11-13]. These systems do away with the spectrometer slit altogether and simply view the source through a rotating dispersive element. In this approach, the measurements taken at different rotation angles of the dispersive element are projective measurements through the data cube and can be tomographically reconstructed. This technique has a very high photon collection efficiency. However, the range of angles over which projections are made is limited by the geometry of the system aperture and the distance to the object. The limited range of angles produces an unsampled region of Fourier space, and an estimate of the data cube that is necessarily inexact. In the tomographic community this Fourier undersampling is known as the missing cone problem, because the unsampled region is a conical volume in Fourier space. There has been significant work on algorithmic approaches for "filling in" this missing information. The most successful has been the method of projection onto convex sets (POCS) [14].

This manuscript considers a completely static, single-shot approach that relies on no complicated optical elements. As a result it is extremely mechanically robust and inexpensive. Like the direct-view methods, our approach does not measure all of the information in the data cube. However, unlike those methods, the lack of information measurement is intentional and has a specifically-designed structure. Our approach draws on the 
recent success of compressive sensing [6-8,15-21]. The ability to solve such an underdetermined problem relies on the properties of "natural" signals—-specifically that they tend to be sparse in some basis other than the naïve Dirac sampling basis.

The sensor design considered in this manuscript derives from our previous work on coded aperture spectrometers [22] and spectral imagers [23]. The basic design for a compressive imaging spectrometer was presented in [24], which considered least gradient and related diffusion algorithms for decompressive spectral image inference. The present manuscript focuses on multiscale decompressive inference algorithms using the dual disperser optical design of [24]. Prior to describing the algorithms, we briefly review the optical design with particular attention to recent advances in physical layer code design.

\subsection{Prototype system and system model}

As described above, the goal is to produce a system with arbitrary wavelength filters on each spatial pixel. In this way, the system can be viewed as a generalization of the Bayer filter pattern that is used in digital color imaging. For hyperspectral imaging, however, interference-based filters, such as those used in the Bayer system are not practical. The large number of spectral channels in a hyperspectral imager requires very narrow spectral features, while the need for high photon collection requires a large number of active channels in any given filter function. Producing filter functions with large numbers of narrow transmission/rejections bands would be cost-prohibitive via interference filters.

In our approach, which we dub the dual-disperser system, the filter functions are produced by a simple aperture code placed between two dispersive elements with equal and opposite effect. A schematic of the system is shown in Fig. 1.

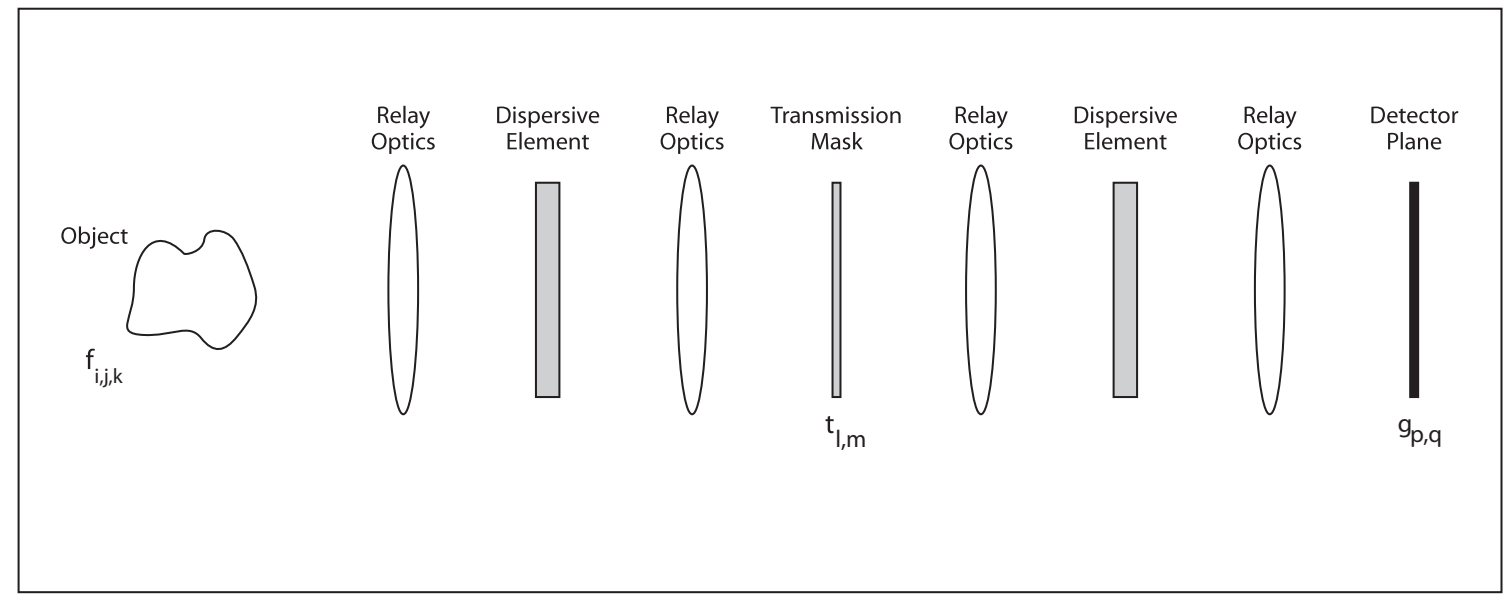

Figure 1. A schematic of the notional imaging system.

In developing a system model, we assume all physical scales are matched to the sampling size of the detector and work in a completely discrete representation. As shown in Fig. 1, the source is taken to have a spectral density $\boldsymbol{f}_{i, j, k}$, where the first, second, and third indices indicate the two spatial and one spectral coordinates, respectively. The light from the source is imaged through the first dispersive element onto an aperture mask with a binary-valued transmission given by $\boldsymbol{t}_{l, m}$. This mask modulates the intensity of the light and the resulting distribution is imaged through a second dispersive element onto a two-dimensional detector array which measures the incident intensity profile $\boldsymbol{g}_{p, q}$. 
To determine the relationship between the detector measurements and the source spectral density, we begin following the propagation of the source through the system. The first dispersive element produces a wavelengthdependent shift of the image on the aperture mask. As a result, the first index of the source (representing the vertical spatial index) becomes a mixture of the spatial and wavelength indices. We can write the spectral density just prior to the aperture mask as $\boldsymbol{f}_{(i+k), j, k}$.

Passage through the aperture mask is represented as multiplication by $\boldsymbol{t}_{l, m}$. The coding plane is then imaged through another dispersive element (with a dispersion equal and opposite to the first dispersive element) onto a two-dimensional detector plane. This undoes the index mixing in $f$ but introduces a similar index mixing in $t$. The intensity pattern recorded (without noise) on the detector can be written as

$$
\begin{aligned}
\boldsymbol{g}_{p, q} & =\sum_{k, l, m, p, q} \boldsymbol{f}_{i, j, k} \boldsymbol{t}_{(l-k), m} \delta[l-i] \delta[m-j] \delta[p-i] \delta[q-j] \\
& =\sum_{k} \boldsymbol{f}_{p, q, k} \boldsymbol{t}_{(p-k), q},
\end{aligned}
$$

where $\delta[a]=\left\{\begin{array}{cc}1, & a=0 \\ 0, & \text { else }\end{array}\right.$. The Kronecker-deltas represent the imaging properties of the optical system, while the sum in $k$ results from that fact that the detector is wavelength insensitive. This measurement can be viewed as the imposition of the $m^{\text {th }}$ row-code along the wavelength direction of $\boldsymbol{f}$. However, we have the added complication that the code is circularly shifted by an amount that depends on the value of the $p$-index.

Thus we have succeeded in our goal of producing highly-structured spectral filter functions on a pixel-bypixel basis, subject only to the circular-shift relationship described above. The next section describes our choice of aperture code (and hence spectral filters).

\subsection{Code design}

To create a system with $M$ spectral channels requires a 1-D code of length $M$. The physical nature of the system produces spectral filters that are all $M$ possible cyclic shifts of the fundamental code. Thus we are led to consider well-conditioned codes that consist of cyclic permutations of a single master codeword. The canonical example of these types of codes are those based on the cyclic S-matrices [25]. For our initial system (and earliest simulations), we drew upon the order-15 cyclic S-matrix with the fundamental codeword "100100011110101". This code provides an overall transmission efficiency of $8 / 15$.

If the coding plane were directly tiled with this pattern, the various filter functions would be implemented on the detector plane in the manner depicted in Figure 2. In this image, the number $k$ denotes the $k^{\text {th }}$ spectral filter function, which corresponds to the fundamental codeword circularly shifted by $k-1$ bits; thus 1 refers to the fundamental codeword "100100011110101", 2 refers to the shifted codeword "001000111101011", etc. The difficulty with this arrangement is that there are no compact regions that contain all of the filter functions.

\begin{tabular}{|l|l|l|l|l|l|l|l|l|l|l|l|l|l|l|l|l|}
\hline 15 & 1 & 2 & 3 & 4 & 5 & 6 & 7 & 8 & 9 & 10 & 11 & 12 & 13 & 14 & 15 & 1 \\
\hline 15 & 1 & 2 & 3 & 4 & 5 & 6 & 7 & 8 & 9 & 10 & 11 & 12 & 13 & 14 & 15 & 1 \\
\hline 15 & 1 & 2 & 3 & 4 & 5 & 6 & 7 & 8 & 9 & 10 & 11 & 12 & 13 & 14 & 15 & 1 \\
\hline
\end{tabular}

Figure 2. Distribution of filter functions that arises from simple tiling of the fundamental codeword. No compact region contains all 15 filters. 
If we instead tile the aperture plane with a unit cell of the form

100100011110101

001111010110010

101011001000111

(three copies of the fundamental codeword, but with circular shifts of five elements between rows) then the filter functions are arrayed on the detector plane as displayed in Figure 3. In this scheme, any $3 \times 5$ region on the detector contains all 15 spectral filters.

\begin{tabular}{|c|c|c|c|c|c|c|}
\hline 5 & 6 & 7 & 8 & 9 & 10 & 11 \\
\hline 15 & 1 & 2 & 3 & 4 & 5 & 6 \\
\hline 5 & 6 & 7 & 8 & 9 & 10 & 11 \\
\hline 10 & 11 & 12 & 13 & 14 & 15 & 1 \\
\hline 15 & 1 & 2 & 3 & 4 & 5 & 6 \\
\hline
\end{tabular}

Figure 3. Distribution of filter functions that arises from the more complicated unit cell. Any $3 \times 5$ region contains all 15 filters.

\section{MULTISCALE SPATIO-SPECTRAL INTENSITY ESTIMATION}

Before presenting our approach to reconstructing the three-dimensional spatio-spectral data cube using the coded measurements described in Section 2, we describe a novel method for removing noise from spatio-spectral data; the solution of the inverse problem described above will then build upon this intensity estimation method.

Assume that we measure noisy observations of a spatially- and spectrally-varying intensity, where the noise is obeys a Poisson distribution associated with photons hitting the detector array. Let $\boldsymbol{f}$ denote the $N \times N \times M$ true three-dimensional intensity, where the first two dimensions correspond to the spatial locations, and the third dimension corresponds to the spectral wavelengths. Our observations (in this section) are thus assumed to be of the form

$$
\boldsymbol{y} \sim \operatorname{Poisson}(\boldsymbol{f}) .
$$

Our goal is to infer $\boldsymbol{f}$ from the measurements $\boldsymbol{y}$ as accurately as possible, effectively utilizing the spectral information contained in the third dimension.

\subsection{Limitations of existing approaches}

The spectral information contained in the third dimension of this data cube conveys important information about color, and, in many applications, important material properties. However, it does not directly lead to improved denoising capabilities. One common mechanism for improving the signal-to-noise ratio is to simply aggregate the observations into spatial bins regardless of their wavelengths. This has two key disadvantages. First, after binning the spectral information is lost and can no longer be used to further the scientific objectives of imagers. Second, while the signal-to-noise ratio in each spatial bin is relatively large, the contrast between spatial locations can be dramatically reduced. This occurs when, for example, two spatial locations have distinctly different spectra, but the total amount of luminosity, aggregated across spectral bands, is similar for the two spatial locations. 
A second approach might involve denoising the image in each spectral band independently. While there exist several effective tools for image denoising and analysis under Poisson noise, this approach ignores the key prior knowledge that the same spatial features (e.g. boundaries between two different material types) exist at every spectral band, even if they are difficult to detect in a given band.

Third, we might consider treating the spectral information as additional dimensions of the data and then perform multidimensional intensity estimation (such as thresholding multidimensional wavelets based on tensor products or pruning a multidimensional tree-based partition of the data hypercube). However, such approaches implicitly assume similar degrees of intensity smoothness in all dimensions, while in fact (a) the structures prevalent in spatial dimensions are rarely the same as structures prevalent in spectral dimensions, and (b) in some cases we may not be able to assume any smoothness between spectral bands.

The different natures of spatial and spectral dimensions were addressed in [26] by using a discrete wavelet transform in the spatial domain and the discrete Fourier transform in the spectral domain. While this is an effective tool in some contexts, it has limited utility for very photon-limited data because the statistical behavior of the coefficients under Poisson noise are difficult to characterize and hence many coefficient denoising rules are subject to inaccuracies.

\subsection{Proposed approach}

Taking advantage of correlations in the data between both wavelengths and spatial locations, the proposed method entails performing hereditary Haar intensity estimation via tree pruning in the spatial dimensions, with each leaf of the resulting unbalanced quad-tree decomposition corresponding to a region of spatially homogeneous spectra.

In particular, we determine the ideal partition of the spatial domain of observations (assumed to be $[0,1]^{2}$ ) and use maximum penalized likelihood estimation to fit a single spectrum to each square in the optimal spatial partition. The space of possible partitions is a nested hierarchy defined through a recursive dyadic partition (RDP) of $[0,1]^{2}$. The optimal partition is selected by merging neighboring squares of (i.e. pruning) a quad-tree representation of the observed data to form a data-adaptive RDP $\mathcal{P}$. Each of the terminal squares in the pruned spatial RDP could correspond to a region of intensity which is spatially homogeneous or smoothly varying (regardless of the regularity or irregularity between the spectral bands). This gives our estimators the capability of spatially varying the resolution to automatically increase the smoothing in very regular regions of the intensity and to preserve detailed structure in less regular regions.

Given a partition $\mathcal{P}, \widehat{\boldsymbol{f}}(\mathcal{P})$ can be calculated by finding the "best" spectrum fit to the observations over each cell in $\mathcal{P}$. In the case where the intensity is not expected to vary smoothly from one spectral band to the next, this can be accomplished by simply computing the mean observed spectrum in each cell of $\mathcal{P}$. However, in many cases we may accurately assume that the intensities in neighboring spectral bands are similar, and so the spectral estimate for a given cell can be computed using penalized likelihood intensity estimation methods. In particular, we sum the observation in each cell for each spectral band, yielding Poisson observations of the aggregate spectrum in each partition cell (denoted $\boldsymbol{y}^{(c)}$ for cell $c \in \mathcal{P}$ ). We then prune an RDP representation of this observed spectrum for each partition cell independently. This procedure, detailed in [27], is highly analogous to the spatial tree pruning described above. Thus for each cell $c$ in partition $\mathcal{P}$, we build and prune a complete RDP of the spectrum. In this case, the spectral RDP takes the form of a binary tree representation, and the model fit to each terminal cell in the spectral RDP is simply the empirical mean of the spectrum across the corresponding 
spectral bands. More formally, for each cell $c$ in a partition $\mathcal{P}$ we compute

$$
\begin{aligned}
& L^{(c)}=\min _{\widetilde{\boldsymbol{f}}^{(c)}}\left\{-\log p\left(\boldsymbol{y}^{(c)} \mid \widetilde{\boldsymbol{f}}^{(c)}\right)+\operatorname{pen}_{1}\left(\widetilde{\boldsymbol{f}}^{(c)}\right)\right\} \\
& \widehat{\boldsymbol{f}}^{(c)}=\underset{\widetilde{\boldsymbol{f}^{(c)}}}{\arg \min }\left\{-\log p\left(\boldsymbol{y}^{(c)} \mid \widetilde{\boldsymbol{f}}^{(c)}\right)+\operatorname{pen}_{1}\left(\widetilde{\boldsymbol{f}}^{(c)}\right)\right\}
\end{aligned}
$$

where $\widetilde{\boldsymbol{f}}^{(c)}$ is constrained to be zero outside cell $c$ and to correspond to a pruned RDP with sample means fit to the leaf nodes, $L^{(c)}$ denotes the penalized likelihood of the estimate in cell $c$,

$$
p\left(\boldsymbol{y}^{(c)} \mid \widetilde{\boldsymbol{f}}^{(c)}\right)=\prod_{(i, j) \in c} \prod_{k=0}^{M-1} \frac{e^{-\tilde{\boldsymbol{f}}_{i, j, k}^{(c)}}\left(\widetilde{\boldsymbol{f}}_{i, j, k}^{(c)}\right)^{y_{i, j, k}}}{y_{i, j, k} !}
$$

denotes the likelihood denotes the likelihood of observing $\boldsymbol{y}^{(c)}$ given the image estimate $\widetilde{\boldsymbol{f}}^{(c)}$ and $\operatorname{pen}_{1}\left(\widetilde{\boldsymbol{f}}^{(c)}\right)$ is the penalty associated with the estimate $\widetilde{\boldsymbol{f}}^{(c)}$. We penalize the estimates according to the number of terminal cells in the pruned binary RDP; the penalties are discussed in detail in [27,28]. The resulting estimate $\widehat{\boldsymbol{f}}^{(c)}$ is referred to as a penalized likelihood estimate (PLE) for cell $c$. The final spatio-spectral estimate is then calculated by finding the partition which minimizes the total penalized likelihood function:

$$
\begin{aligned}
\widehat{\mathcal{P}} & \equiv \underset{\mathcal{P}}{\arg \min }\left\{\sum_{c \in \mathcal{P}} L^{(c)}+\operatorname{pen}_{2}(\mathcal{P})\right\} \\
\widehat{\boldsymbol{f}} & \equiv \widehat{\boldsymbol{f}}(\widehat{\mathcal{P}})=\left\{\sum_{c \in \widehat{\mathcal{P}}} \widehat{\boldsymbol{f}}^{(c)}\right\},
\end{aligned}
$$

where $\operatorname{pen}_{2}(\mathcal{P})$ is a penalty on the size of the spatial partition which can be used to encourage spatial smoothing beyond that induced by the spectral penalty pen $_{1}$. This supplemental penalty is not necessary in many contexts, but is particularly helpful when no regularization or smoothing is desired in the spectral dimension (i.e. when $\left.\operatorname{pen}_{1}(\cdot)=0\right)$.

This approach is similar to the image estimation method described in [28,29], with the key distinction that the proposed method forces the spatial RDP to be the same at every spectral band. A sample such partition is displayed in Figure 4. This constraint makes it impossible for the method to perform spatial smoothing at some spectral bands but not others. In other words, when a tree branch is pruned in the proposed framework, it means partition cells are merged in every spectral band simultaneously at the corresponding spatial location. This approach is effective because an outlier observation in one spatio-spectral voxel may not be recognized as such when spectral bands are considered independently, but may be correctly pruned when the corresponding spectrum is very similar to spatially nearby spectra.

The accuracy of these estimates can be augmented by a process called cycle-spinning, or averaging over shifts, resulting in translation-invariant (TI) estimates [30]. Cycle-spinning was derived in the context of undecimated wavelet coefficient thresholding in the presence of Gaussian noise, but is difficult to implement efficiently in the case of tree-pruning methods. The above multiscale tree-pruning methods can be modified to produce the same effect by averaging over shifts, but the increase in quality comes at a high computational cost. Novel computational methods [29], however, can be used to yield TI-Haar tree pruning estimates in $O\left(N^{2} M \log N \log M\right)$ time for an $N \times N \times M$ data cube. 


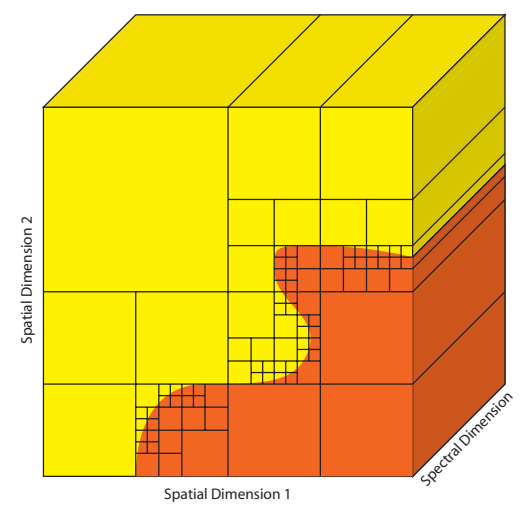

Figure 4. Sample partition of a spatio-spectral data cube. The spatial partition is the same at each spectral band, making it impossible for the estimation method to perform spatial smoothing at some spectral bands but not others.

\subsection{Denoising experimental results}

As described above, the proposed method exploits spatial homogeneities while simultaneously taking advantage of correlations between neighboring spectral bands. This significantly reduces over-smoothing across spatial boundaries and edges while improving the reconstruction of each spectrum. To test the effectiveness of the proposed method, we develop a phantom using a color image of peppers, as displayed in Figure 5. For each pixel in this image, we define a spectrum in our phantom data cube which corresponds to the color in the image. This results in a $256 \times 256 \times 64$ data cube, with a mean intensity (photon count) of 7.04 per voxel.

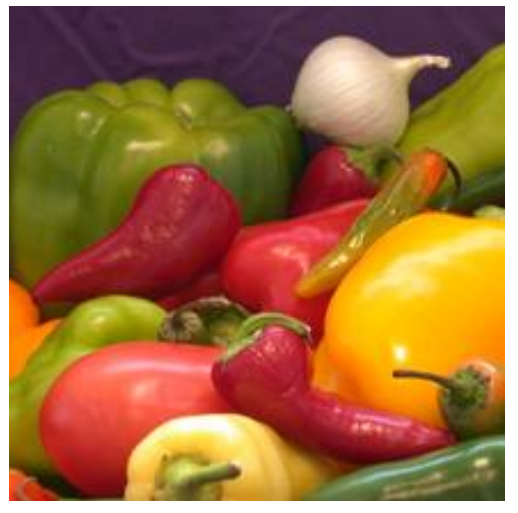

(a)

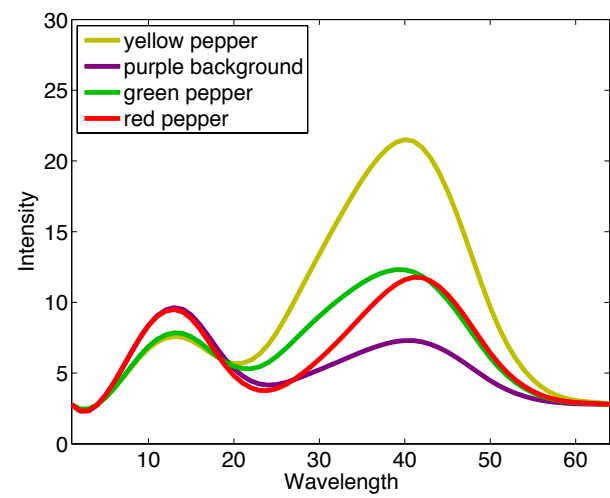

(b)

Figure 5. Test data set. (a) Image used to generate $256 \times 256 \times 16$ spatio-spectral data cube. (b) Representative selection of generated spectra in different image regions. Wavelength units are normalized so each spectral band corresponds to one wavelength unit.

The spatially-varying intensities for three representative spectral bands (one from the red portion of the simulated spectrum ( $16^{\text {th }}$ spectral band), one green $\left(24^{\text {th }}\right.$ spectral band), and one blue ( $53^{\text {rd }}$ spectral band)) are displayed in Figure 6(a), (f), and (k). Note the faint contrast between some of the features. Noisy observations in the same three spectral bands are displayed in Figure 6(b), (g), and (l). Several faint or low-contrast features are not easily discernible by the eye due to the low photon counts. In this simulation study, the penalty term in (2) was multiplied by a scalar weight to improve empirical performance. The weight was not selected to minimize any particular error metric, but rather to yield generally accurate reconstructions. 
If we were to reconstruct this data cube by performing hereditary TI-Haar image estimation [29] on each spectral band, we would achieve the results displayed in Figure 6(c), (h), and (m); the mean squared error associated with this imaging-based data cube estimate, denoted $\widehat{\boldsymbol{f}}_{I}$, is $\left\|\boldsymbol{f}-\widehat{\boldsymbol{f}}_{I}\right\|^{2} /\|\boldsymbol{f}\|^{2}=5.22 \cdot 10^{-3}$. (While the images may appear oversmoothed, decreasing the weight on the penalty term results in strong noisy artifacts and increases the MSE significantly.) Spatio-spectral analysis offers dramatic advantages over processing individual spectral bands independently, as shown in Figure 6(d), (i), and (n). These results were generated by setting pen $_{1}$ proportional to the number of leaves in the full spatio-spectral RDP $\widehat{\mathcal{P}}$ and $\operatorname{pen}_{2}$ to zero (i.e. the total penalty depends on both the smoothness in the spectral dimension and in the spatial dimensions). The mean squared error associated with this spatio-spectral data cube estimate, denoted $\widehat{\boldsymbol{f}}_{S 1}$, is $\left\|\boldsymbol{f}-\widehat{\boldsymbol{f}}_{S 1}\right\|^{2} /\|\boldsymbol{f}\|^{2}=$ $3.26 \cdot 10^{-3}$, significantly lower than the MSE for $\widehat{\boldsymbol{f}}_{I}$. This simulation result shows several spatial features, including faint boundaries and small-scale inhomogeneities, which are present in the true and spatio-spectrally estimated intensities but which are difficult or impossible to extract from the noisy observations or independently processed spectral bands. Similarly encouraging results are possible even without smoothing in the spectral dimension, which is accomplished by setting $\operatorname{pen}_{1}$ to zero and $\mathrm{pen}_{2}$ proportional to the number of leaves in the spatial RDP (i.e. the total penalty is independent of the degree of smoothness in the spectral dimension). These results are displayed in Figure 6(e), (j), and (o), and the mean squared error associated with this data cube estimate, denoted $\widehat{\boldsymbol{f}}_{S 1}$, is $\left\|\boldsymbol{f}-\widehat{\boldsymbol{f}}_{S 2}\right\|^{2} /\|\boldsymbol{f}\|^{2}=4.02 \cdot 10^{-3}$. Finally, Figure 7 shows four representative spectra and their estimates, further demonstrating the strength of the proposed method.

\section{SPECTRAL IMAGE RECONSTRUCTION}

The above multiscale method for spatio-spectral denoising can now be used to help reconstruct the spectral image indirectly measured by the camera described in Section 2. In particular, we can model our observations as

$$
\boldsymbol{x} \sim \operatorname{Poisson}(\boldsymbol{g})=\operatorname{Poisson}(\boldsymbol{H} \boldsymbol{f}),
$$

where $\boldsymbol{f}$ is the vectorized $256 \times 256 \times 15$ spatio-spectral data cube we wish to reconstruct, $\boldsymbol{H}$ is the operator induced by the spectral imaging system as in (1), and $\boldsymbol{x}$ is the vectorized $256 \times 256$ collection of measurements. Note that $\boldsymbol{H}$ has many more columns $(983,040)$ than rows $(65,536)$, making this a very underdetermined problem.

To solve this challenging inverse problem, we seek a solution $(\widehat{\boldsymbol{f}})$ which is both a good match to the data $(\boldsymbol{x})$ and sparse. In particular, we solve the following optimization problem:

$$
\widehat{\boldsymbol{f}}=\underset{\widetilde{\boldsymbol{f}} \in \mathcal{F}}{\arg \min }\{-\log p(\boldsymbol{x} \mid \boldsymbol{H} \widetilde{\boldsymbol{f}})+\operatorname{pen}(\widetilde{\boldsymbol{f}})\}
$$

where $\mathcal{F}$ is the collection of estimators corresponding to a pruned spatio-spectral tree (denoted $\widetilde{\mathcal{P}}$ ) as described in Section 3, and $\operatorname{pen}(\widetilde{\boldsymbol{f}})=\sum_{c \in \widetilde{\mathcal{P}}} \operatorname{pen}_{1}\left(\widetilde{\boldsymbol{f}}^{(c)}\right)+\operatorname{pen}_{2}(\widetilde{\mathcal{P}})$; this penalty is proportional to the number of cells in the pruned RDP and hence the penalty term encourages solutions with small numbers of leaves (i.e. sparse).

The use of sparsity to solve challenging and ill-posed inverse problems has received widespread attention recently $[6-8,19,20]$. The objective function proposed in (4) in particular is similar to those proposed in $[19,20]$, in that we seek a solution accurately represented by a small number of cells in an RDP (i.e. sparse). We compute the solution to this problem using an Expectation-Maximization algorithm, which in this case is a regularized version of the Richardson-Lucy algorithm [31-33]. The method consists of two alternating steps:

\section{Step 1:}

$$
\boldsymbol{y}^{(t)}=\widehat{\boldsymbol{f}}^{(t)} \cdot \times \boldsymbol{H}^{T}\left(\boldsymbol{x} \cdot / H \widehat{\boldsymbol{f}}^{(t)}\right),
$$

where.$\times$ and.$/$ denote element-wise multiplication and division, respectively. 


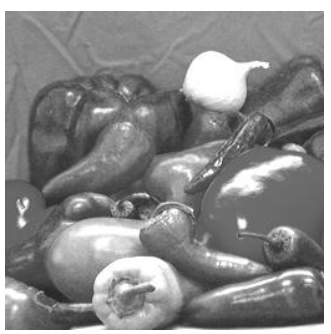

(a)

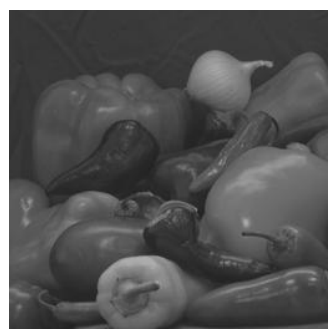

(f)

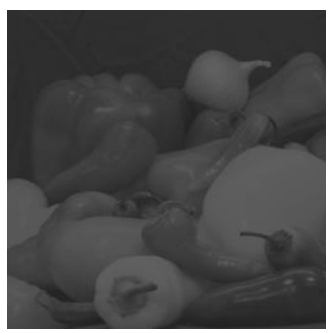

(k)

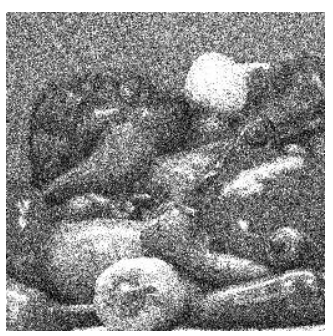

(b)

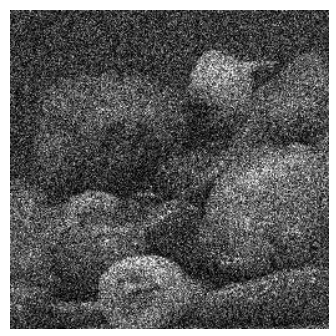

(g)

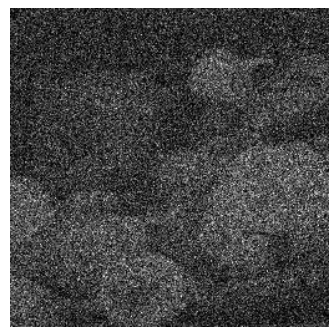

(1)

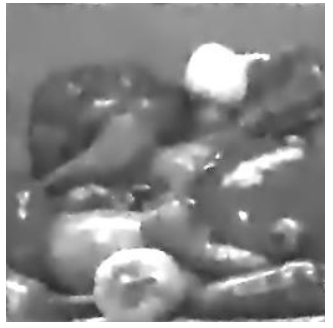

(c)

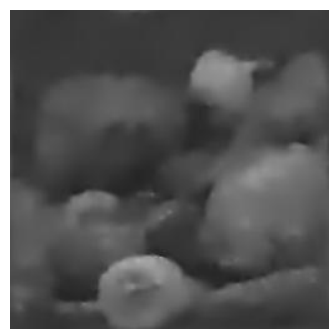

(h)

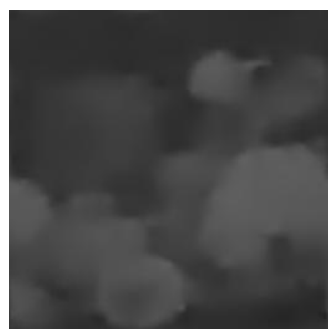

(m)

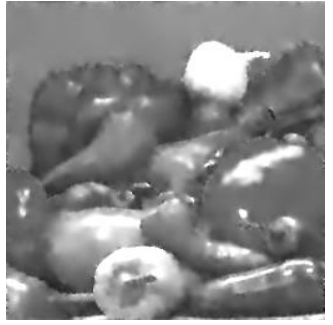

(d)

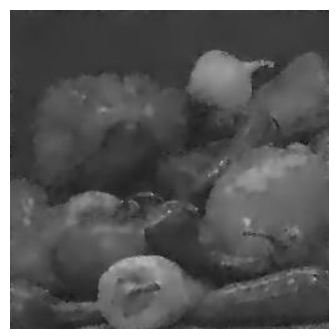

(i)

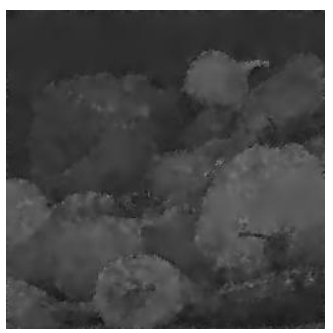

(n)

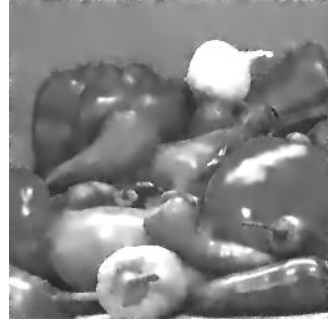

(e)

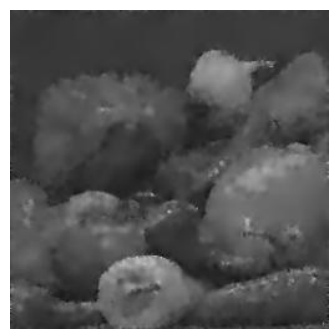

(j)

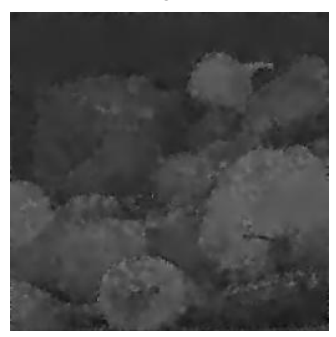

(o)

Figure 6. Spatio-spectral denoising results. (a) Spatial variation of intensity in $16^{\text {th }}$ spectral band (red). (b) Noisy observations, with an average of 7.04 photon count per voxel. (c) Result after using tree-pruning to denoise the image in each

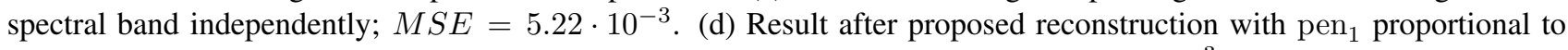
the number of leaves in the full spatio-spectral partition and $\mathrm{pen}_{2}=0 ; \mathrm{MSE}=3.26 \cdot 10^{-3}$. (e) Result after proposed reconstruction with pen ${ }_{1}=0$ (i.e. no smoothing in the spectral direction) and $\mathrm{pen}_{2}$ proportional to the number of leaves in the partition $\widehat{\mathcal{P}}$; MSE $=4.02 \cdot 10^{-3}$. (f) Spatial variation of intensity in $24^{\text {th }}$ spectral band (red). (g) Noisy observations. (h) Result after using tree-pruning to denoise the image in each spectral band independently. (i) Result after proposed

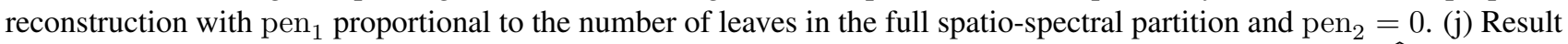

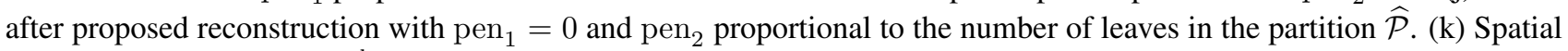
variation of intensity in $53^{\text {rd }}$ spectral band (red). (l) Noisy observations. (m) Result after using tree-pruning to denoise the

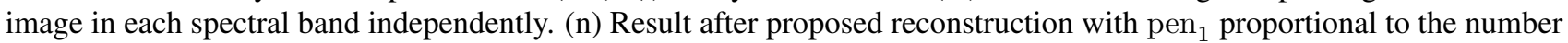
of leaves in the full spatio-spectral partition and $\operatorname{pen}_{2}=0$. (o) Result after proposed reconstruction with pen $_{1}=0$ and pen $_{2}$ proportional to the number of leaves in the partition $\widehat{\mathcal{P}}$.

Step 2: Compute $\widehat{\boldsymbol{f}}^{(t+1)}$ by denoising $\boldsymbol{y}^{(t)}$ according to (3) as described in Section 3.

In the experiments below, the method is initialized with $\widehat{\boldsymbol{f}}^{(0)}=\boldsymbol{H}^{T} \boldsymbol{x}$ and terminated when $\| \widehat{\boldsymbol{f}}^{(t+1)}-$ $\widehat{\boldsymbol{f}}^{(t)}\left\|_{2}^{2} /\right\| \widehat{\boldsymbol{f}}^{(t)} \|_{2}^{2}<3 \cdot 10^{-6}$.

\section{SPECTRAL IMAGE RECONSTRUCTION RESULTS}

This method is used to reconstruction a spatio-spectral data cube from simulated measurements modeled after the camera described in Section 2. For this experiment, we generated a phantom spatio-spectral data cube similar 


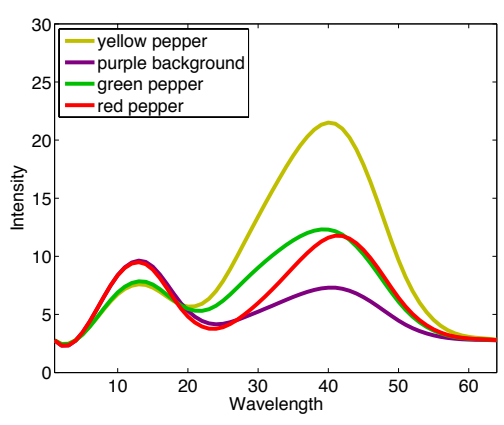

(a)

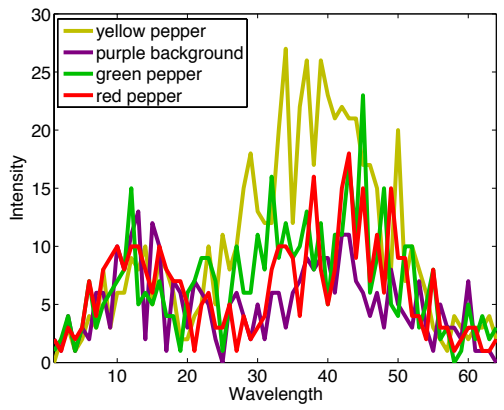

(b)

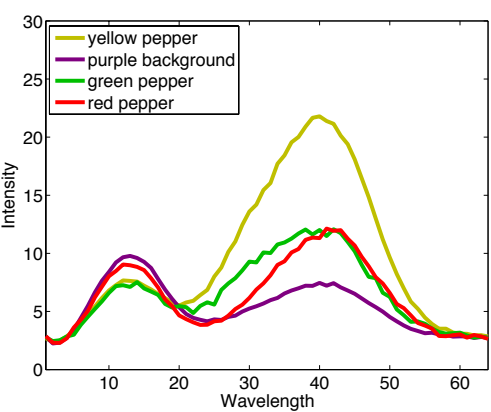

(c)

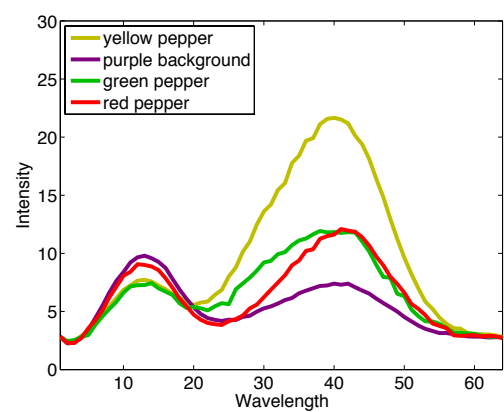

(d)

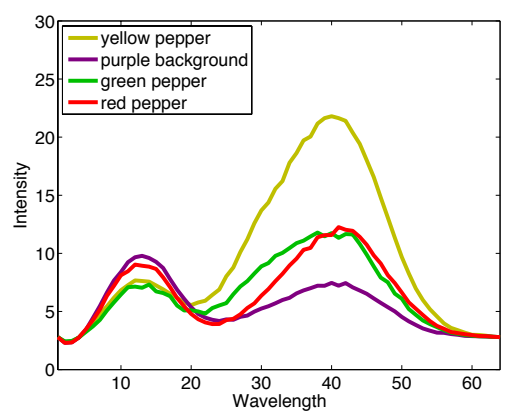

(e)

Figure 7. Spatio-spectral analysis results. (a) True spectra at five different spatial locations. (b) Observed spectra at same locations. (c) Estimated spectra at same locations after performing intensity estimation on each spectral band independently, resulting in $\widehat{\boldsymbol{f}}_{I}$. (d) Estimated spectra at same locations after performing proposed spatio-spectral intensity estimation, resulting in $\widehat{\boldsymbol{f}}_{S 1}$. (e) Estimated spectral at same locations after performing two-stage approximate estimation, resulting in $\widehat{\boldsymbol{f}}_{S 2}$.

to the one used in Section 3, but with only 15 (instead of 64) spectral bands. The intensities in several spectral bands, the color projection of the data cube, and several representative spectra for this phantom are displayed in the first row of Figure 9. The $256 \times 256$ noisy simulated observations are displayed in Figure 8; in this experiment, the mean photon count per pixel in $\boldsymbol{x}$ is 1,534 .

The result of the proposed reconstruction method is displayed in the second row of Figure 9. For this reconstruction, we do not perform smoothing in the spectral dimension (i.e. $\mathrm{pen}_{1}=0$ and $\mathrm{pen}_{2}$ is proportional to the number of leaves in the partition $\widehat{\mathcal{P}}$ ) because of the small number of spectral bands and the potential for significant spectral inhomogeneities at this spectral resolution. The reconstructed spectral image computed using the multiscale denoising method described in Section 3 has an MSE of $3.42 \cdot 10^{-2}$, while the reconstructed spectral image computed without any regularization (i.e. the conventional Richardson-Lucy reconstruction) has an MSE of $4.64 \cdot 10^{-2}$ and significantly more artifacts.

\section{DISCUSSION}

This paper demonstrates that compressive spectroscopic imaging measurements combined with reconstruction methods based on efficient representations of spatio-spectral data cubes can yield accurate reconstructions in the presence of noise, despite the limited number of measurements collected. To our knowledge, this work is the first application of compressive sensing ideas to spectroscopic imaging, and demonstrates the potential of that framework to ease conventional limitations on spatial and spectral resolution imposed by the size of the system's detector array. 


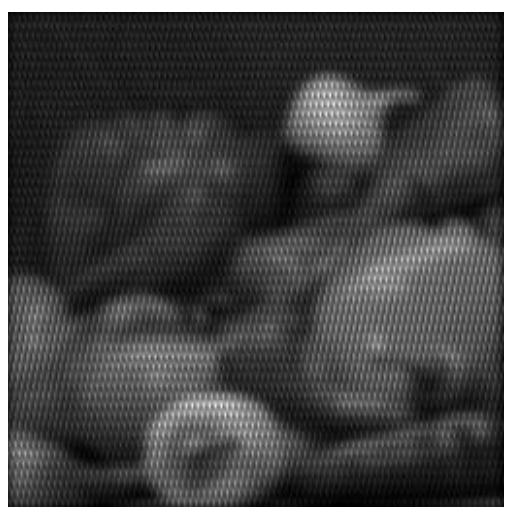

Figure 8. Simulated noisy observations of peppers spatio-spectral data cube collected by proposed coded spectral imaging system.

The proposed imaging system, unlike conventional systems, does not collect a unique measurement for each voxel in the desired spectral image. Rather, it uses a transmissive mask to encode spectral information into a relatively small number of projections of the spectral image. The spectral information is then extracted from these compressive, noisy measurements using a computation method which seeks a "sparse" spectral image which is a good match (in a log likelihood sense) to the measured data. Sparsity is measured using a novel tree-based representation of spatio-spectral data cubes.

In the proposed method, we build a quad-tree decomposition of the data cube in the spatial dimension, so that each leaf node of the tree corresponds to a spectrum. We then prune back this tree using a penalized likelihood criterion. In contrast to processing the "image" at each spectral band independently, this method imposes the constraint that spatial smoothing be the same across all spectral bands.

In the context of image estimation, multiscale methods based on hereditary Haar wavelets are near minimax optimal reconstruction techniques for piecewise smooth images [27]. This suggests that the proposed method potentially has similar optimality characteristics for some classes of marked Poisson processes. Theoretical analysis of the proposed method is an important component of our ongoing work. In addition, the masks used to encode spectral information in the proposed system are cyclic S-matrices [25], which have high transmission efficiency and yield the encouraging results presented in Section 5. However, it may be possible to design alternative masks or spectral encoding strategies, subject to the constraints imposed by the spectral imaging system design, which increase the potential accuracy of the reconstructed data cubes. Investigation of efficient code design strategies for compressive spectral imaging is an additional important area of ongoing work.

\section{ACKNOWLEDGEMENTS}

The authors would like to thank Nikos Pitsianis and Timothy Schultz for valuable discussions and suggestions.

\section{REFERENCES}

1. P. A. Townsend, J. R. Foster, J. R. A. Chastain, and W. S. Currie, "Application of imaging spectroscopy to mapping canopy nitrogen in the forests of the central appalachian mountains using hyperion and aviris," Geoscience and Remote Sensing, IEEE Transactions on 41(6), pp. 1347-1354, 2003.

2. W. Smith, D. Zhou, F. Harrison, H. Revercomb, A. Larar, A. Huang, and B. Huang, "Hyperspectral remote sensing of atmospheric profiles from satellites and aircraft," in Proc. SPIE, 4151, pp. $94-102,2001$.

3. R. P. Lin, B. R. Dennis, and A. O. B. (Eds.), The Reuven Ramaty High-Energy Solar Spectrscopic Imager (RHESSI) - Mission Description and Early Results, Kluwer Academic Publishers, Dordrecht, 2003. 


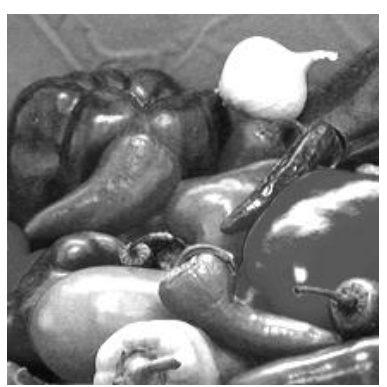

(a)

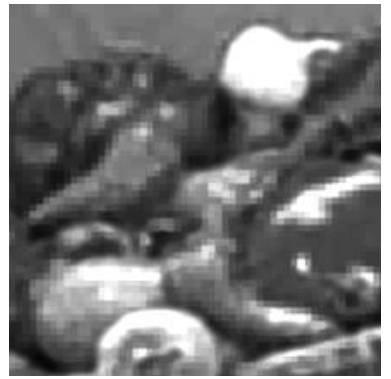

(e)

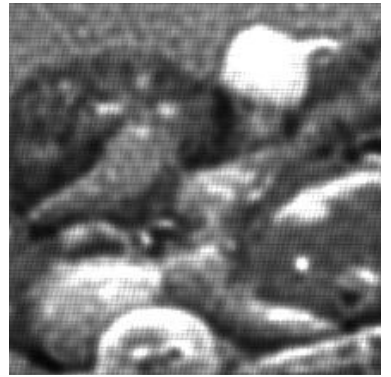

(i)

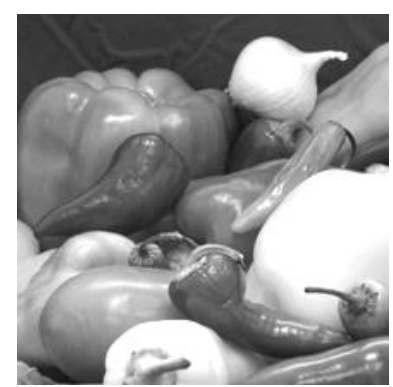

(b)

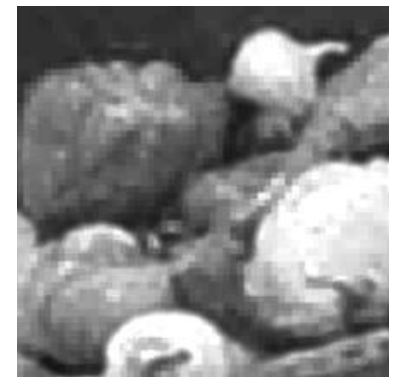

(f)

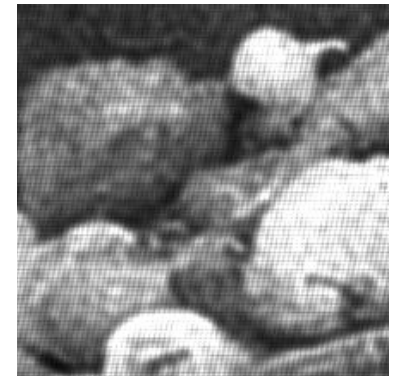

(j)

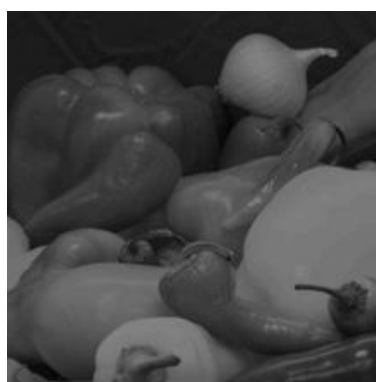

(c)

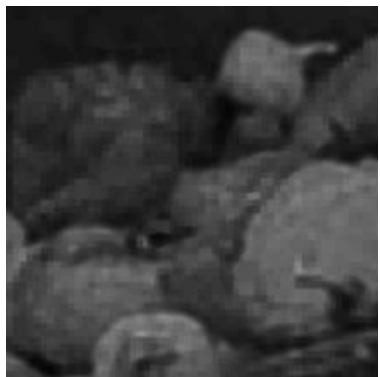

(g)

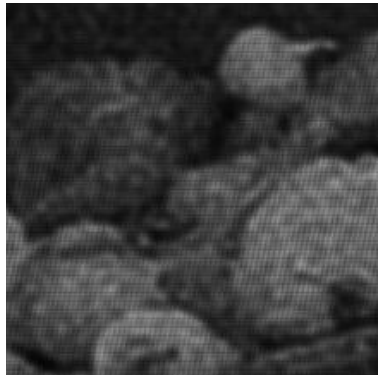

(k)

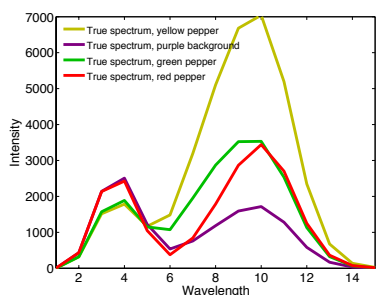

(d)

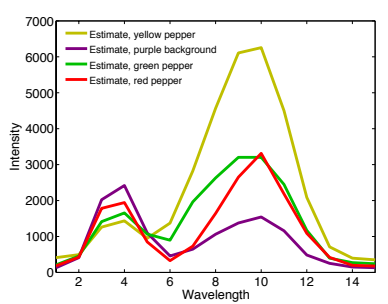

(h)

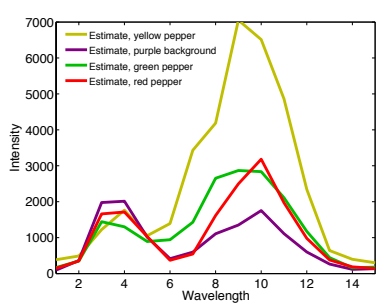

(l)

Figure 9. Spatio-spectral reconstruction results. (a) True intensity in $4^{\text {th }}$ spectral band. (b) True intensity in $8^{\text {th }}$ spectral band. (c) True intensity in $12^{\text {th }}$ spectral band. (d) Representative spectra from true spatio-spectral data cube. (e) Estimated intensity in $4^{\text {th }}$ spectral band, computed using reconstruction method described above with multiscale spatio-spectral regularization; $M S E=3.42 \cdot 10^{-2}$. (f) Estimated intensity in $8^{\text {th }}$ spectral band, computed using reconstruction method described above with multiscale spatio-spectral regularization. (g) Estimated intensity in $12^{\text {th }}$ spectral band, computed using reconstruction method described above with multiscale spatio-spectral regularization. (h) Representative spectra from estimated spatio-spectral data cube, computed using reconstruction method described above with multiscale spatiospectral regularization. (i) Estimated intensity in $4^{\text {th }}$ spectral band, computed using reconstruction method described above with no regularization; $M S E=4.64 \cdot 10^{e-2}$. (j) Estimated intensity in $8^{\text {th }}$ spectral band, computed using reconstruction method described above with no regularization. (l) Estimated intensity in $12^{\text {th }}$ spectral band, computed using reconstruction method described above with no regularization. (l) Representative spectra from estimated spatio-spectral data cube, computed using reconstruction method described above with no regularization.

4. W. Denk, J. Strickler, and W. Webb, "Two-photon laser scanning fluorescence microscopy," Science 248(4951), pp. 73-76, 1990.

5. R. Schultz, T. Nielsen, J. Zavaleta, R. Ruch, R. Wyatt, and H. Garner, "Hyperspectral imaging: A novel approach for microscopic analysis," Cytometry 43, pp. 239 - 247, 2001.

6. E. Candès, J. Romberg, and T. Tao, "Robust uncertainty principles: Exact signal reconstruction from highly incomplete frequency information," IEEE Transactions on Information Theory 52(2), pp. 489 - 509, 2006. 


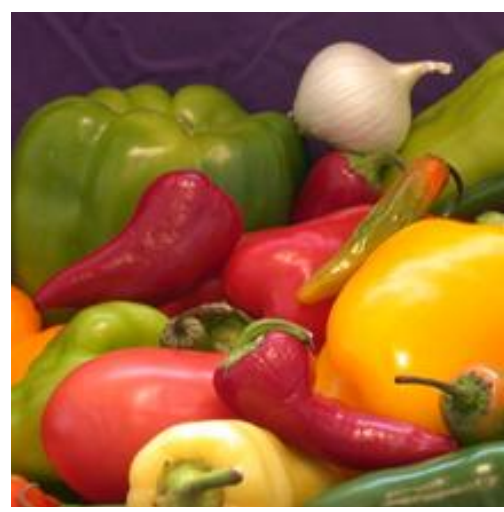

(a)

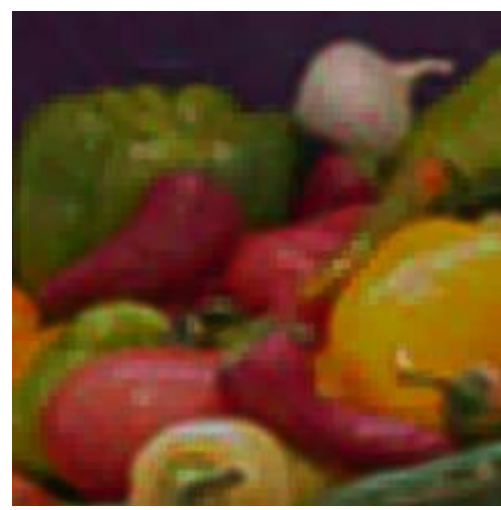

(b)

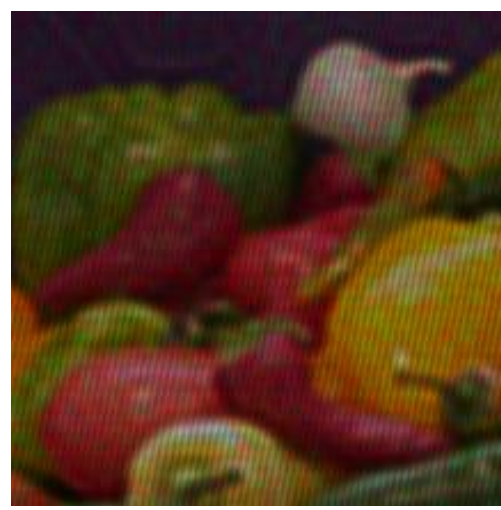

(c)

Figure 10. Spatio-spectral reconstruction results, color projections. (a) Color projection of true spatio-spectral data cube. (b) Color projection of estimated spatio-spectral data cube, computed using reconstruction method described above with multiscale spatio-spectral regularization. (c) Color projection of estimated spatio-spectral data cube, computed using reconstruction method described above with no regularization.

7. E. Candés and T. Tao, "Near optimal signal recovery from random projections: Universal encoding strategies." To be published in IEEE Transactions on Information Theory. http://www.acm.caltech.edu/ emmanuel/papers/OptimalRecovery.pdf, 2006.

8. D. Donoho, “Compressed sensing," IEEE Transactions on Information Theory 52(4), pp. 1289-1306, 2006.

9. C. Snively, G. Katzenberger, and J. Lauterbach, "Fourier-transform infrared imaging using a rapid-scan spectrometer," Opt. Lett. 24, pp. 1841-1843, 1999.

10. A. Wuttig and R. Riesenberg, "Sensitive Hadamard transform imaging spectrometer with a simple MEMS," Proc. SPIE 4881, pp. 167-178, 2003.

11. M. Descour and E. Dereniak, "Computed-tomography imaging spectrometer: experimental calibration and reconstruction results," Appl. Opt. 34, pp. 4817-4826, 1995.

12. P. Bernhardt, "Direct reconstruction methods for hyperspectral imaging with rotational spectrotomography," J. Opt. Soc. Am. A 12, pp. 1884-1901, 1995.

13. J. Mooney, V. Vickers, M. An, and A. Brodzik, "High-throughput hyperspectral infrared camera," J. Opt. Soc. Am. A 14, pp. 2951-2961, 1997.

14. A. Brodzik and J. Mooney, "Convex porjections algorithm for resotration of limited-angle chromotomographic images," J. Opt. Soc. Am. A 16, pp. 246-257, 1999.

15. M. A. Neifeld and P. Shankar, "Feature-specific imaging," Applied Optics 42(17), pp. 3379-3389, 2003.

16. H. S. Pal and M. A. Neifeld, "Multispectral principal component imaging," Optics Express 11(18), pp. 2118-2125, 2003.

17. Y. Tsaig and D. Donoho, "Extensions of compressed sensing," tech. rep., Stanford University, 2004.

18. N. Pitsianis, D. Brady, and X. Sun, "Sensor-layer image compression based on the quantized cosing transform," Proc. SPIE 5817, pp. 250-257, 2005.

19. J. Haupt and R. Nowak, "Signal reconstruction from noisy random projections." To be published in IEEE Transactions in Information Theory. http://www.ece.wisc.edu/ nowak/infth.pdf, 2006.

20. E. Candès, J. Romberg, and T. Tao, "Stable signal recovery from incomplete and inaccurate measurements," Communications on Pure and Applied Mathematics 59(8), pp. 1207-1223, 2006.

21. D. Brady, M. Gehm, N. Pitsianis, X. Sun, and P. Potuluri, "Compressive sampling strategies for integrated microspectrometers," Proc. SPIE, 2006.

22. M. Gehm, S. McCain, N. Pitsianis, D. Brady, P. Potuluri, and M. Sullivan, "Static 2D aperture coding for multimodal multiplex spectroscopy," Appl. Opt. 45, pp. 2965-2974, 2006. 
23. M. E. Gehm and D. J. Brady, "High-throughput hyperspectral microscopy," Three-Dimensional and Multidimensional Microscopy: Image Acquisition and Processing XIII 6090(1), SPIE, 2006.

24. D. J. Brady and M. E. Gehm, "Compressive imaging spectrometers using coded apertures," Visual Information Processing XV 6246(1), SPIE, 2006.

25. M. O. Harwitt and N. J. A. Sloane, Hadamard Transform Optics, Academic Press, 1979.

26. I. Atkinson, F. Kamalabadi, and D. L. Jones, "Wavelet-based hyperspectral image estimation," 2, pp. 743-745, 2003.

27. R. Willett and R. Nowak, "Multiscale poisson intensity and density estimation." Submitted to IEEE Trans. Info. Th., 2005.

28. E. Kolaczyk and R. Nowak, "Multiscale likelihood analysis and complexity penalized estimation," Annals of Stat. 32, pp. 500-527, 2004.

29. R. Willett and R. Nowak, "Fast multiresolution photon-limited image reconstruction," in Proc. IEEE Int. Sym. Biomedical Imaging - ISBI '04, (15-18 April, Arlington, VA, USA), 2004.

30. M. Lang, H. Guo, J. E. Odegard, C. S. Burrus, and R. O. Wells, "Noise reduction using an undecimated discrete wavelet transform," IEEE Signal Processing Letters 3(1), pp. 10-12, 1996.

31. W. Richardson, "Bayesian-based iterative method of image restoration," J. Opt. Soc. of Am. 62, pp. 55-59, 1972.

32. L. B. Lucy, "An iterative technique for the rectification of observed distributions," Astron. J. 79, pp. 745-754, 1974.

33. R. Willett and R. Nowak, "Platelets: a multiscale approach for recovering edges and surfaces in photon-limited medical imaging," IEEE Transactions on Medical Imaging 22(3), pp. 332-350, 2003. 